\title{
Effectiveness, safety and patients' satisfaction of a new treatment for facial ageing with topical autologous platelet rich plasma mixed with hyaluronic acid after microneedling: results of a single centre preliminary study
}

\begin{abstract}
Introduction: Platelet Rich Plasma (PRP) and Hyaluronic acid (HA) have been proven to be effective in skin rejuvenation. The aim of this single Centre pilot study was to demonstrate the effectiveness and the safety of a new mixture of PRP and HA applied topically after microneedling to rejuvenate chrono- and photo-damaged facial skin.

Methods: ten patients aged $43.3 \pm 7.7$ years, Glogau $\geq \mathrm{II}$ were enrolled in the study and underwent three treatments each. After preparation, PRP was mixed with non-cross-linked HA and administered topically after microneedling of the facial skin. The Wrinkle Severity Rating Scale (WSRS) and the Global Aesthetic Improvement Scale (GAIS) were used respectively by a Plastic Surgeon and by the patients to assess effectiveness of the technique.

Results: No early and/or late complications were observed after the treatments. Both WSRS and GAIS score improved with statistical significance before and after the procedures and comparing the first and second, second and third and, most of all, first and third topical application.

No significant differences were found comparing subgroups of patients based on age $(\leq 49$ or $\geq 50$ ), smoking habits (smokers and non-smokers), patients who practised sports or not, patients taking drugs for pre-existing disease or not, summer sun-exposed patients or not.

Conclusion: This new mixture of PRP-HA with the minimally invasive method of application (topical after microneedling) has proven to be effective and free of side effects in our series. Further studies with a greater number of enrolled patients are needed in order to confirm our results.
\end{abstract}

Volume 3 Issue 4 - 2019

\author{
Teresa Salerno,' Emanuele Dragonetti, ${ }^{2}$ Sara \\ Angelone, ${ }^{3}$ Enrico Baldessari, ${ }^{\prime}$ Michele de \\ Nuntiis $\mathrm{MD}^{1}$ \\ 'Eur Plastic Med Clinic, Italy \\ ${ }^{2}$ Rome American Hospital, Italy \\ ${ }^{3}$ Ministry of Health, Italy
}

Correspondence: Teresa Salerno, MD Eur Plastic Med Clinic, Via Aldo Ballarin 2, 00142 Rome, Italy, Email teresa_salerno@yahoo.com

Received: July 10, 2019 | Published: July 16, 2019

\section{Introduction}

Platelet-rich Plasma (PRP) is an autologous concentration of human platelets in a small volume of plasma, obtained by the centrifugation of the patients' own blood. Platelets contain Growth Factors (GF) and mediators in their alpha-granules, including Plateletderived GF (PDGF), Transforming GF (TGF), Vascular Endothelial GF (VEGF) and Insulin-like GF (IGF). These GF stimulate tissue remodelling and are associated with enhanced healing through the attraction of macrophages, up regulation of collagen synthesis and promotion of tissue regeneration. ${ }^{1,2}$

It is widely used indifferent clinical settings and adopted in Dermatology and Plastic Surgery because it is considered a "natural" way to reduce skin damage due to chrono- and photo-ageing. PRP is hypothesized to improve skin quality, texture and tone via injection and/or in combination with microneedling technology. In vitro studies have suggested a role for PRP in increasing collagen expression, matrix remodelling proteins, fibroblast proliferation and differentiation into myofibroblasts. ${ }^{3}$

Skin changing associated with ageing are due to the loss of dermal proteins such as collagen and elastin (resulting in the modification of extracellular matrix), hyaluronic acid (HA) and proteoglycans, resulting in changes of the extracellular matrix, reduction of epidermis hydration, provoking the formation of wrinkles, fine lines, colour changes, dryness and decreased elasticity. Among the factors playing an important role in facial aging are exposure to ultraviolet (UV) light, smoking habits and alcohol consumption, which, together with genetic factors, can alter the rate of skin aging. Several options are available to prevent or treat skin aging, including surgery, skin resurfacing and the utilization of injectable dermal fillers. HA, in particular, is widely used thanks to its high biocompatibility (being a component of the extracellular matrix and being present in large amounts in human tissues) and its ability to reduce wrinkles (either superficial or deep) and general skin aging.

Microneedling is a treatment in which the needles are rolled over the skin, determining healing micro punctures with micro wounds in the epidermis and dermis. This injury triggers the inflammatory cascade and stimulates collagen and elastic fibers production, resulting in skin remodeling. In patients with atrophic acne scars, therapy can lead to improved scar appearance, skin texture, and patient satisfaction. ${ }^{4,5}$

To date there are no published data about the combination of PRP and HA applied topically on the face to correct the signs of ageing, neither papers where topical PRP is associated to microneedling to rejuvenate the facial skin. The only published data in which PRP is 
used topically to improve face appearance in healthy volunteers is from Yuksal et al, who obtained a significant improvement in the patients' auto-evaluation rate, but the medical assessment showed a significant difference only for firmness-sagging. ${ }^{6}$

\section{Aim of the study}

This single Centre pilot study aims to evaluate the clinical improvements, the effectiveness and the safety, in terms of shortand long-term side effects, of combined topic PRP and HA in after microneedling for the management of ageing of the face.

\section{Materials and methods}

\section{Patients selection}

Ten consecutive patients referred to our Clinic for facial rejuvenation were enrolled in the study. Inclusion criteria were absence of systemic chronic disease, Platelet value in the blood cell count higher than $150.000 / \mathrm{mL}$, absence of skin disease or acute skin infections, such as Herpes simplex-I, Glogau wrinkle scale rate $\geq 2 .^{7}$

Exclusion criteria were pregnancy and lactation, autoimmunitary disease, immunosuppressive treatments, haematological disease, other local treatments, such as botulin toxin, filler, bio revitalization before this procedure, intake of aspirin and FANS in the 4 weeks before the treatment, previous surgical procedures of the face. All the patients were subjected to full history taking (including lifestyle), general and local examination and photographing of the face before and after each session. An informed consent was obtained from each patient for treatment, photo-taking and use for scientifical purposes.

\section{Prp-Hamix preparation}

Ten $\mathrm{ml}$ of whole blood were collected and PRP was obtained as described by Yuksal and coworkers ${ }^{6}$ Then a fix amount of $5 \mathrm{ml}$ of PRP was taken and mixed with $20 \mathrm{mg}$ of non cross- linked low molecular weight HA (1200 Da) through two luer-lock syringes. The mixture was used immediately.

\section{Dermaroller procedure}

The face was disinfected with hydrogen peroxide, then microneedling was performed using a dermaroller with a 540 fine titanium needles, length $0,75 \mathrm{~mm}$, that was passed over the stretched skin in vertical, horizontal, and both diagonal directions for about 10 passes in each direction, always by the same operator, creating pores on the skin through which the mixture of PRP-HA was supposed to pass and act. Self-limited erythema and minimal oozing occurred at the needling puncture areas on the skin easily controlled by salinesoaked gauze.

Topical application of the complex PRP-HA was performed at this step by the same operator, with a standardized massage technique. The patients were forbidden to wash or rub their faces to avoid rinsing the unabsorbed part of PRP-HA combination still on the skin for 24 hours; past this term it was possible to wash the part and apply the usual cosmetic products routinely used by the patients. Furthermore, they were invited to avoid sun exposure and to use sun screens daily with a sunburn protecting factor of 30 or more.

\section{Clinical scores}

A clinical score before and after the administration was assigned to each patient by two blinded independent Plastic Surgeons. The score used was the Wrinkle Severity Rating Scale (WSRS), which provides a score ranging from a minimum of 1 (absence of wrinkles) to a maximum of 5 points (extremely deep and long wrinkles, which worsen the appearance of the face, wrinkles deep V 2 to $4 \mathrm{~mm}$ with stretched skin). ${ }^{8}$

In addition, patients were asked to self-assign a score of degree of the improvement perceived, using the Global Aesthetic Improvement Scale (GAIS scale), which has a variable score from 0 (worsening compared to before treatment) to a maximum of 4 (extremely improved, optimal cosmetic result). ${ }^{9}$

\section{Statistical analysis}

Data were analyzed using the SPSS calculation system. Quantitative variables were described in terms of mean \pm standard deviation, range (minimum and maximum). To establish the statistical significance of the comparisons between two independent means, the t-student test was used, and a value was considered statistically significant if $\mathrm{p}<0.05$.

\section{Results}

This was a single Centre pilot study, conducted on 10 consecutive patients who turned to our Clinic with the request for facial rejuvenation. Patients mean age was 43,3 $\pm 7,7$ years (31-56). Time elapsed between subsequent administrations averaged $4.3 \pm 0.47$ weeks (4-5). We investigated the patients' lifestyle, asking questions about sun exposure habits, smoking, sports activities, any previous and current disease and pharmacological therapies: 5 out of 10 patients $(50 \%)$ were smokers, with an average consumption of 10 cigarettes per day. All but 2 patients $(20 \%)$ practiced sports, with a weekly average time of 3.6 hours per week. Eight patients $(80 \%)$ reported summer sun exposure with an average exposure period of $40 \pm 23.3$ days, all after sunscreen application.

Three patients (30\%) were on drug therapies: 2 were administered Thyroid Substitutive treatment, one was on antidepressive therapy.

Clinical features of the patients are summarized in Table 1. No early side effects (edema, anaphylaxis, persistent erythema, burning, itching, flaking), defined as occurring in the first 48hours after the application,were observed, except minimal erythema and oozing, or late (defined as side effects that occurred after 48hours, such as hematomas, skin pigmentation changes, pain). The patients reported just a minimal discomfort due to the use of the dermaroller.

Table I Clinical Features of the patients

\begin{tabular}{ll}
\hline $\mathrm{N}^{\circ}$ patients & 10 \\
$\mathrm{M} / \mathrm{F}$ & $0 / 10$ \\
Mean age (years) & $42,3 \pm 7.7$ \\
Smoker & $5(50 \%)$ \\
Mean $\mathrm{n}^{\circ}$ sigarettes/day & 10 \\
Sportive pts & 8 \\
Mean hrs sport/week & 3,6 \\
Sun-exposed pts & 8 \\
Mean exposure/year (days) & $40 \pm 23,3$ \\
Drugs-intaking pts $\mathrm{N}^{\circ}$ & $3(30 \%)$ \\
Thyroid Hormones & 2 \\
Antidepressive treatment & $\mathrm{I}$ \\
\hline
\end{tabular}


The WSRS score showed a statistically significant improvement between the pre- and post-applicationscore in all the three sessions, achieving the higher significative difference comparing the beginning of the study (pre-I) to the end of the study (post -III) with a p value of 0.0001 (Table 2).

Table 2 Comparison of WSRS score before and after subsequent applications

\begin{tabular}{llll}
\hline I PRE & vs & POST & P 0.02 \\
II PRE & vs & POST & P 0.04 \\
IIIPRE & vs & POST & P 0.000 I
\end{tabular}

The GAIS score showed the same trend, reaching a $\mathrm{p}$ value at the end of the study equal to 0.0001 (Table 3). However, all the patients had already reported an improvement in the texture and skin uniformity of the face after the first treatment.

Table 3 Comparison of GAIS score before and after subsequent applications

\begin{tabular}{llll}
\hline I & vs & II & $\mathrm{P} 0.03$ \\
II & vs & III & $\mathrm{P} 0.000$ I \\
I & vs & III & $\mathrm{P} 0.0000$ I \\
\hline
\end{tabular}

Due to the small number of patients, it was difficult to identify subgroups based on different anamnestic features, in order to assess any statistically significant differences between the subgroups.

As far as possible to evaluate, there were no statistically significant differences before and after the treatment dividing patients according to age (49 or $\geq 50$ ), to smoking habits (smokers and non-smokers), between those who smoked more or less than 20cigarettes a day, to sport activity (sportive or sedentary), between who practiced more than 4 hours of sport per week and who practiced less than 4 , and to drug intake.

Figure 1 shows the trend ina patient enrolled in the study: a fiftytwo year old female, smoker, sportive ( 2 hour/week), in whom brilliant results in terms of reduction of nasolabial and frontal wrinkles, tear trough and in skin appearance is clearly evident (Figure 2).

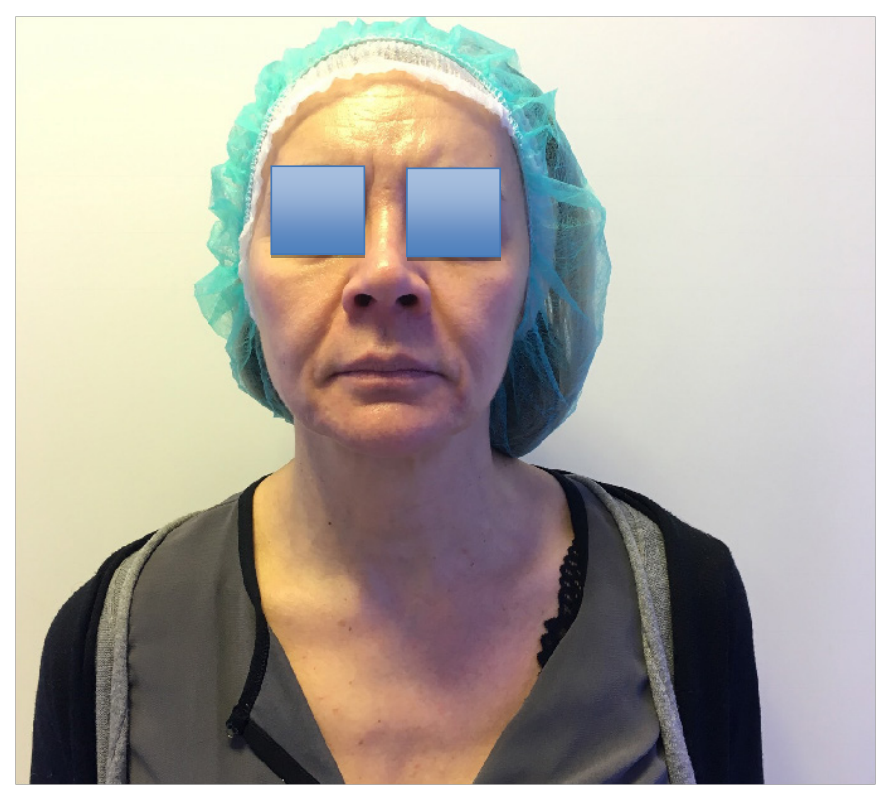

Figure I fifty-two year old smoker patient.A: before the treatment.
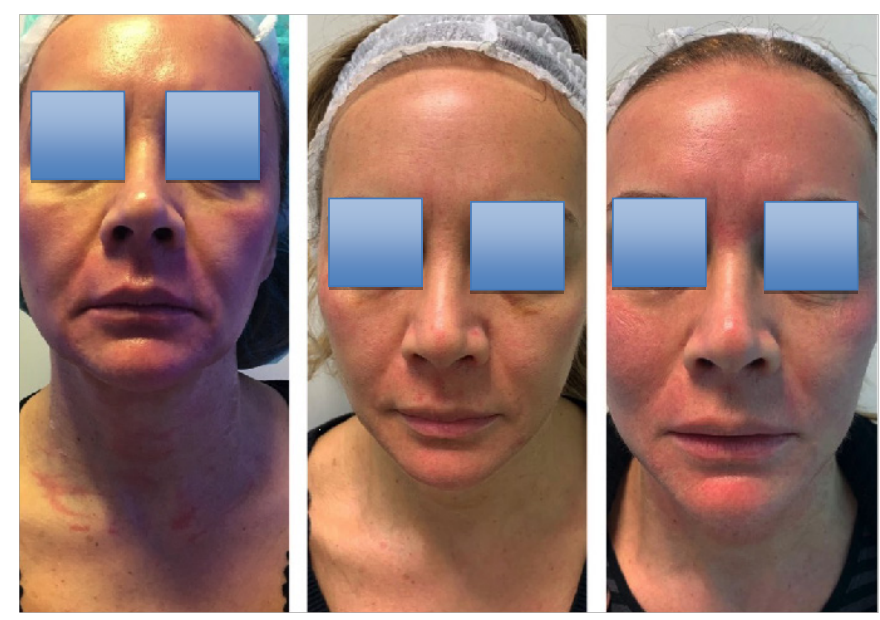

Figure 2 B: after the first treatment; C: after the second treatment; D: after the third treatment.

\section{Discussion}

The aim of our study was the evaluation of the effectiveness and safety of a topically administered new mixture of PRP-HA, after microneedling,for facial skin rejuvenation in a group of patients from our Clinic.

We demonstrated a significant efficacy of topical PRP-HA in the subsequent applications, achieving highly significant differences comparing the scores before the first and after the third and last treatment. To date no previous studies using topical associated PRPHA have been reported in the literature for this purpose. The only study that describes the use of topical PRP in improving the aspect of facial skin is from Yuksel et al: PRP was applied thrice at 2 weeks intervals on the face of 10 healthy volunteers and applied with a dermaroller on the forehead, malar area and jaw and injected into the wrinkles of crow's feet.

A grade on a five-point scale(for general appearance, skin firmnesssagging, wrinkle state and pigmentation disorder of the face) was autoassigned by the patients and by three dermatologists before each PRP procedure and 3 months after the last PRP procedure. These Authors found a statistically significant difference regarding the general appearance, skin firmness-sagging and wrinkle state according to the patients' auto-evaluation, whereas there was significant difference only for the skin firmness-sagging according to the assessment of the dermatologists. They concluded that PRP application could be considered as an effective procedure for facial skin rejuvenation. ${ }^{6}$

Our study confirmed their results in terms of effectiveness of topical PRP for facial ageing, with the difference that we used added HA to PRP to enrich the potential role of the mixture and two different valued scales of evaluation for physicians and patients: the improvement was significant both in terms of patients satisfaction and medical evaluation.

Several studies described the effectiveness of microneedling and PRP in treating acne scars, topical or intradermal injection administration: in this case the combination treatment appears to be effective in cosmetic outcomes, post procedural downtime and patient satisfaction. ${ }^{10}$

The lack of standardization of the technique of preparation of PRP, the number of sessions required, the time interval that must elapse

Citation: Salerno T, Dragonetti E, Angelone S, et al. Effectiveness, safety and patients' satisfaction of a new treatment for facial ageing with topical autologous platelet rich plasma mixed with hyaluronic acid after microneedling: results of a single centre preliminary study.J Dermat Cosmetol. 20I9;3(4):94-98. DOI: $10.15406 / j d c .2019 .09 .00121$ 
between administrations, the long-term follow-upmakes it difficult to compare the different studies already present in the literature. This is even more relevant in our case, given the novelty of the product and our administration technique. PRP preparation method influences the final number of Platelet and their activation. ${ }^{6,11}$ Further studies are needed to determine the optimal method and procedure.

The use of PRP in the treatment of chrono-and photo-aging has been widely discussed in the literature confirming its effectiveness. A recent review confirmed the role of platelets, growth factors, leukocytes and plasma as fibroblast proliferation agents. These cells are able to migrate and proliferate in the tissue surrounding a wound and to deposit granulation tissue, which minimizes the scar; their antiaging effectiveness is linked to the ability to synthesize collagen and elastin, which are the main components of the ECM of the dermis. The function of leukocytes, their ability to repair, to "dialogue" with other cells and to orchestrate different outcomes, is receiving increasing attention in Regenerative Medicine.

The most recent review emphasizes the ability of PRP to act in face lifting, in improving facial volumes in areas with signs of emptying, in rejuvenation, regeneration and reconstruction of the skin, in the improvement of wrinkles, in the ability to stimulate hair growth, to increase the vitality of the follicle and its survival rate, prevent apoptosis, increase and prolong the anagen phase of growth, improve the caliber of the stem, their shine and elasticity. Furthermore the use in the contouring (filler) and in the reduction of the melasma is also taken into consideration. ${ }^{2,12}$

We did not observe any short- and/or long- term side effects, the only discomfort was due to the dermaroller, but it was transient and self-controlled. We have also demonstrated that life-style does not affect the results of the treatment, this can be interpreted as a strong point, as the results can be found also in smokers, sedentary and sunexposed patients.

In a very recent paper from Dhurat $\mathrm{S}$ and coworkers, a tattoo dye pigment was used to examine dermal penetration after microneedling in a single patient: in their experience the low molecular pigment failed to penetrate the infundibulum, so they speculate that GF, which are larger molecules, are not likely to be delivered to their target tissue with this technique. They conclude that topical PRP applied with microneedling cannot exert a beneficial physiological effect. ${ }^{13}$

These data are in contrast with previous and equally recent publications, in which microneedling was successfully used to deliver drugs, genes, proteins, RNA, hormones and vaccines, that are considerably much heavier than our mixture. Transdermal administration is considered noninvasive of course, can be selfpracticed, avoids first-pass metabolism, so in these papers is highly indicated to administer even high molecular weight molecules. ${ }^{14,15-22}$

The strength of our work lies in the simplicity of the mixture preparation and in the minimally invasive route of administration, that avoids needle use, more accepted by the patients and free from undesirable effects, while its weakness is represented by the small number of patients enrolled so far and by the shortness of follow-up to demonstrate the permanence of the results achieved.Therefore, the study should be considered as an initial pilot study to test the method, the efficacy and the safety of the mixture.

It will be absolutely necessary to increase the number of patients and sessions to validate our results and a more lasting follow-up to assess the long-term efficacy of the protocol.

\section{Conclusion}

The novel PRP-HA approach for the treatment of facial aging administered topically showed good tolerance and efficacy. The results obtained in the small sample we examined encourages a larger sample study, since a highly significant difference was demonstrated between the pretreatment and after three treatments in both medical and patient satisfaction quantitative evaluation, performed with standardized scales. Therefore, new scenarios are opening up in chrono- and photoaging treatment strategies with a low-invasive procedural protocol and low clinical risk.

\section{Acknowledgments}

None.

\section{Conflicts of interest}

Author declares that there is no conflict of interest.

\section{Funding}

None.

\section{References}

1. Adrian DK Le, Lawrence Enweze, Malcolm R, et al. Platelet Rich Plasma. Clin Sports Med. 2019;38(1):17-44.

2. Ibrahim MK, Ibrahim SM, Salem AM. Skin microneedling plus plateletrich plasma versus skin microneedling alone in the treatment of atrophic post acne scars: a split face comparative study. J Dermatolog Treat. 2018;29(3):281-286.

3. Z-Hye Lee, Sammy Sinno, Grace Poudrier BA, et al. Platelet rich plasma for photodamaged skin: A pilot study. $J$ Cosmetol Dermatol. 2019;18(1):77-83

4. Sparavigna A, Tenconi B, Giori AM, et al. Evaluation of the efficacy of a new hyaluronic acid gel on dynamic and static wrinkles in volunteers with moderate aging/photoaging. Clini Cosmet Investig Dermatol. 2019:1281-1290

5. Hashim PW, Levy Z, Cohen JL, et al. Microneedling therapy with and without Platelet Rich Plasma. Cutis. 2017; 99(4):239-242.

6. Yuksel E, Sahin G, Aydin F, et al. Evaluation of effects of platelet-rich plasma on human facial skin. J Cosmet Laser Ther. 2014;16(5):206-208.

7. Glogau RG. Aesthetic and anatomic analysis of the ageing skin. Semin Cutan Med Surg. 1996;15(3):134-138.

8. Flynn TC, Carruthers A, Carruthers J, et al. Validated assessment scales for the upper face. Dermatol Surg. 2012;38:309-319.

9. Narins RS, Brandt F. Leyden Lorenc ZP. A randomized, double-blind, multicenter comparison of the efficacy and tolerability of Restylane versus Zyplast for the correction of nasolabial folds. Dermatol Surg. 2003;29(6):588-595.

10. Schoenberg E, O Connor M, Wang JV, et al. Microneedling and PRP for acne scars: A new tool in our arsenal. J Cosmet Dermatol. 2019.

11. Shilpa Dastikop, Santoshi MN, SushruthKamoji. To compare different methods of preparationof platelet rich plasma (PRP) and to analyse the correlation between initial CBC and final yield of PRP. $J$ Dermat Cosmetol. 2019;3(3):89-92.

12. Cho JW, Kim SA, Lee KS. Platelet-rich plasma induces increased expression of G1 cell cycle regulators, type I collagen, and matrix metalloproteinase-1 in human skin fibroblasts. Int $J \mathrm{Mol} \mathrm{Med}$. 2012:29(1):32-36. 
13. Dhurat S, Situm M, Kovacevic M. Mission impossible: dermal delivery of Growth Factors via microneedling. Dermatologic Therapy. 2019;32(3):e1287.

14. Pandey PC, Shuklas S, Skoog SA, et al. Current advancements in transdermal biosensing and targeted drug delivery. Sensor (Basels) 2019;19(5): pii: E1028.

15. Liw, ZhouX, Yang F, et al. Microneedled-based transdermal drug delivery systems: a review. J Biochemical Nanotechnol. 2017;13(12):1581-1597.

16. Leheta T, El Tawdy A, Abdel Hay R, et al. Percutaneous collagen induction versus full-concentration trichloroace- tic acid in the treatment of atrophic acne scars. Dermatol Surg. 2011;37(2):207-216.

17. Fabbrocini G, Fardella N, Monfrecola A, et al. Acne scar- ring treatment using skin needling. Clin Exp Dermatol. 2009;34(8):874-879.

18. Zeitter S, Sikora Z, Jahn S, et al. Microneedling: matching the results of medical needling and repetitive treatments to maximize potential for skin regeneration. Burns. 2014;40(5):966-973.
19. El-Domyati M, Abdel-Wahab H, Hossam A. Microneedling combined with Platelet-Rich Plasma or Trichloroacetic acid peeling for management of acne scarring: a split face clinical and histological comparison. J Cosmet Dermatol. 2018;17(1):73-83.

20. Kim DH, Je YJ, Kim CD et al. Can platelet-rich plasma be used for skin rejuvenation? Evaluation of effects of plate- let-rich plasma on human dermal fibroblast. Ann Dermatol. 2011;23(4):424-431.

21. Devereaux J, Nurgali K, Kiatos D, et al. Effects of platelet-rich plasma and platelet-poor plasma on human dermal fibroblasts. Maturites. 2018;117:34-44.

22. Elghblawi E. Platelet-rich plasma, the ultimate secret for youthful skin elixir and hair growth triggering. J Cosmet Dermatol. 2018;17(3):423430 .

Citation: Salerno T, Dragonetti E, Angelone S, et al. Effectiveness, safety and patients' satisfaction of a new treatment for facial ageing with topical autologous platelet rich plasma mixed with hyaluronic acid after microneedling: results of a single centre preliminary study.J Dermat Cosmetol. 20I9;3(4):94-98. 